\title{
A Report on Bilaterally Elongated Superior Cervical Ganglion
}

\author{
Lavanya Rajmohan ${ }^{1}$, Sulochana Sakthivel ${ }^{2}$, Suman Verma ${ }^{2}$, Aravindhan Kishore ${ }^{3}$ \\ Junior Resident, ${ }^{2}$ Associate Professor, ${ }^{3}$ Professor and Head, Department of Anatomy. Jawaharlal Institute of Postgraduate Medical Education and \\ Research. Puducherry
}

\section{Abstract}

Superior cervical ganglion (SCG), the largest of the three cervical sympathetic ganglia, is formed by the fusion of first four cervical ganglia. Bilaterally elongated superior cervical ganglion was observed in a female cadaver during dissection. On the right side, it was 63.74 $\mathrm{mm}$ long and $5.75 \mathrm{~mm}$ wide and on the left side, it was $62.88 \mathrm{~mm}$ and $5.84 \mathrm{~mm}$ respectively. Histological analysis of the ganglion done with toluidine blue staining confirmed the structure of sympathetic ganglion. Superior cervical ganglion is the preferred ganglion for sympathetic block in conditions like trigeminal neuralgia, atypical facial pain,and post-herpetic neuralgia. Even though superior cervical ganglion has been reported to be at the safest location, thetension of sympathetic trunk while retracting the carotid sheath during surgeries might result in Horner's syndrome. Furthermore, a large ganglion may also be confused with deep cervical lymph nodes or retropharyngeal mass during imaging studies. Understanding the variant anatomy of the superior cervical ganglion might serve as a guide for imaging studies, cervical spine surgeries, and sympathetic block.

Keywords: Sympathetic ganglion, vertebral ganglion, sympathetic block.

Corresponding Author: Dr. Sulochana Sakthivel, M.S., Asssociate professor, Department of Anatomy, Jawaharlal Institute of Postgraduate Medical Education and Research, Puducherry.

Received: April 2019

Accepted: May 2019

\section{Introduction}

Cervical sympathetic chain extends from the base of the skull to the neck of the first rib. It has three ganglia namely superior, middle and inferior. Cranially, it continues as internal carotid nerve and caudally, the inferior cervical ganglion may fuse with the first thoracic ganglion to form cervicothoracic/stellate ganglion. Furthermore, an intermediate ganglion might exist along the sympathetic chain, also known as the vertebral ganglion (VG). The superior cervical ganglion (SCG), the largest of the three, is about $2.5-3 \mathrm{~cm}$ length and formed by the fusion of upper four cervical ganglia. It is located at the retrostyloid space, dorsal to styloid curtain muscle and carotid sheath, bounded by posterior belly of digastric and sternocleidomastoid laterally, transverse process of $\mathrm{C} 2$ and $\mathrm{C} 3$ vertebra posteriorly, and by the pharynx medially. ${ }^{[1]}$

The superior cervical ganglionic block is done for diagnostic as well as therapeutic purposes. It is preferred in conditions like trigeminal neuralgia, atypical facial pain, post-herpetic neuralgia and vasospasm after subarachnoid hemorrhage. ${ }^{[2]}$ The risk of injury of the cervical sympathetic chain is not uncommon during surgeries like the anterior or anterolateral approach of cervical spine, or radical neck dissection. ${ }^{[3,4]} \mathrm{A}$ large SCG could also be mistaken for a metastatic retropharyngeal lymph node. ${ }^{[5]}$ Though SCG has been reported to be at the safest location, tension of sympathetic trunk while retracting the carotid sheath during surgeries, might result in Horner's syndrome. ${ }^{[6]}$ Understanding of morphology of SCG is essential in preventing such complications.

\section{Case Report}

Table 1: Morphometric measurements of the superior cervical ganglion.

\begin{tabular}{|l|l|l|l|}
\hline \multicolumn{2}{|l|}{ Dimensions of SCG } & Right (mm) & Left (mm) \\
\hline Length & 63.72 & 62.88 \\
\hline Width & 5.75 & 5.84 \\
\hline \multirow{2}{*}{ Thickness } & Upper & 2.8 & 1.8 \\
\cline { 2 - 4 } & Middle & 1.06 & 1.72 \\
\cline { 2 - 4 } & Lower & 1.86 & 3.08 \\
\hline Distance from the midline & 11.92 & 11.85 \\
\hline $\begin{array}{l}\text { Distance from the medial } \\
\text { border of the longus capitus } \\
\text { muscle }\end{array}$ & Lying over it & 2.8 \\
\hline
\end{tabular}

During dissection of a 68-year old female cadaver, elongated SCG was observed bilaterally with the absence of middle cervical ganglion (MCG). The course of the cervical sympathetic chain was studied after retraction of the carotid artery medially. The SCG had a long course extending from the base of the cranium to the level of C6 vertebra. On the right side, the SCG was $63.72 \mathrm{~mm}$ long and $5.75 \mathrm{~mm}$ wide [Figure 1]. On the left side, the above ganglion was 62.88 $\mathrm{mm}$ long and $5.84 \mathrm{~mm}$ wide [Figure 2]. Thickness of the SCG in its upper, middle and lower part and the distance of SCG from the midline as well as longus capitus muscle were 
also measured [Table 1]. The SCG gave off grey rami communicantes to $\mathrm{C} 1$ to $\mathrm{C} 6$ spinal nerves, branches to the common carotid artery, laryngopharyngeal branches and communicating branches to the vagus nerve on both the sides. MCG was absent on both sides whereas the VG was observed unilaterally on the left side. The inferior cervical ganglion fused with first thoracic ganglion to form stellate ganglion on both sides. Histological analysis of the upper, middle and lower part of SCG, with toluidine blue staining, revealed multipolar neurons with eccentrically placed nuclei and prominent nucleoli in all the three parts.

Table 2: Dimensions of the superior cervical ganglion as reported in the literature.

\begin{tabular}{|c|c|c|c|c|}
\hline Study & $\begin{array}{ll}\begin{array}{l}\text { Number } \\
\text { specimens }\end{array} & \text { of } \\
\end{array}$ & $\begin{array}{l}\text { Length } \\
(\mathrm{mm})\end{array}$ & $\begin{array}{l}\text { Width } \\
(\mathrm{mm})\end{array}$ & $\begin{array}{l}\text { Thickness } \\
(\mathrm{mm})\end{array}$ \\
\hline Kiray et al $^{[8]}$ & 24 ( 12 cadavers) & $27.6 \pm 4.6$ & $8.2 \pm 1.4$ & $3.2 \pm 0.8$ \\
\hline Saylam et al. ${ }^{[6]}$ & 40 (20 cadavers) & $\begin{array}{l}33 \pm 6.2 \\
\text { Maximum } 45.7\end{array}$ & $8.1 \pm 2.8$ & - \\
\hline Yin et al. ${ }^{[7]}$ & 32 cadavers & $\begin{array}{l}\text { Right- } 32.2 \pm 5.3 \\
\text { Left- } 31.9 \pm 5\end{array}$ & $\begin{array}{l}\text { Right- } 7.2 \pm 1.1 \\
\text { Left- } 7 \pm 11.2 \\
\end{array}$ & $\begin{array}{l}\text { Right- } 2.7 \pm 0.3 \\
\text { Left- } 2.7 \pm 0.3 \\
\end{array}$ \\
\hline Mitsuoka et al..$^{[10]}$ & $\begin{array}{l}72 \\
\text { (54 cadavers) }\end{array}$ & $\begin{array}{l}\text { Male: } \\
\text { Right- } 27.62 \pm 3.8 \\
\text { Left- } 28.26 \pm 4.2 \\
\text { Female: } \\
\text { Right- } 26.87 \pm 2.8 \\
\text { Left- } 22.88 \pm 5.3\end{array}$ & $\begin{array}{l}\text { Right- } 8.05 \pm 1.5 \\
\text { Left- } 7.83 \pm 1.7 \\
\text { Right- } 8.55 \pm 1.4 \\
\text { Left- } 8.41 \pm 1.7\end{array}$ & $\begin{array}{l}\text { Right- } 3.67 \pm 1.1 \\
\text { Left- } 3.51 \pm 0.86 \\
\text { Right- } 3.72 \pm 0.86 \\
\text { Left- } 4.02 \pm 0.84\end{array}$ \\
\hline
\end{tabular}

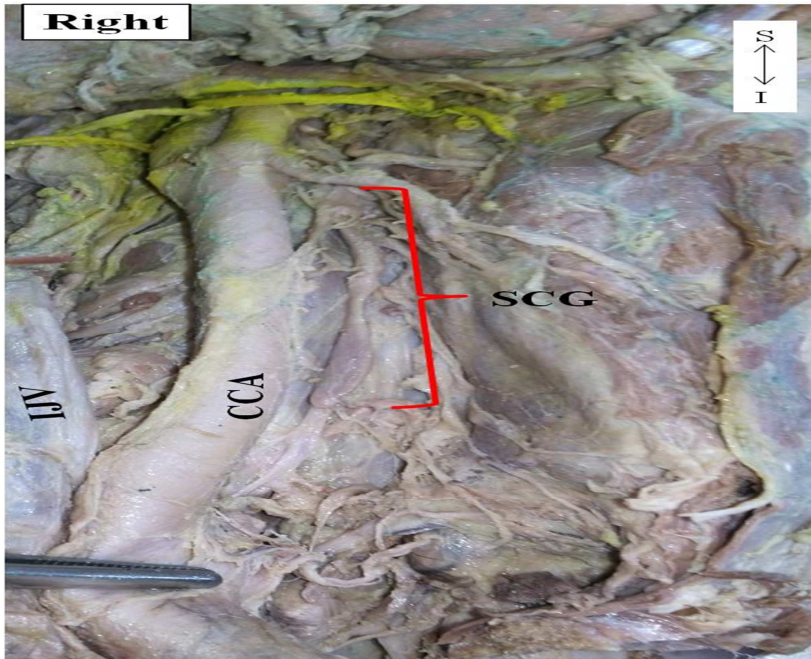

Figure 1: Right superior cervical ganglion (SCG). Common carotid artery (CCA) and internal jugular vein (IJV) are reflected towards right side. S-Superior; I-Inferior.

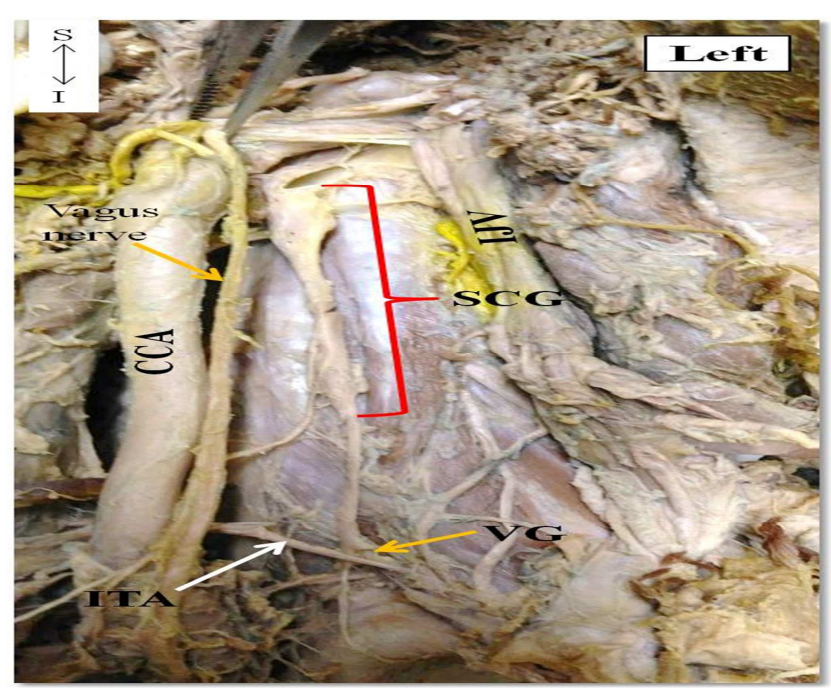

Figure 2: Left superior cervical ganglion (SCG). Common carotid artery (CCA) is reflected medially towards the midline. IJV - Internal jugular vein; S-Superior; I-Inferior.

\section{Discussion}

SCG extends from the cranial base to the $\mathrm{C} 2$ or $\mathrm{C} 3$ vertebral level or at $\mathrm{C} 4$ level. ${ }^{[3,6,7]}$ Saylem et al., reported the presence of SCG in all specimens with a maximum size of $45.7 \mathrm{~mm}$ whereas MCG was observed in $48 \%$ of the cases and double MCG in $10 \%$ of the cases. ${ }^{[6]}$ In a study by Kiray et al., VG was observed in the absence of MCG in $20.8 \%$ of the cases whereas all the four ganglia including VG were observed in $12.5 \%{ }^{[8]}$ Yin et al., observed VG bilaterally in four cadavers and unilaterally in one, on the left side. ${ }^{[7]}$ Dimensions of the SCG as reported by various studies in the literature are given in [Table 2].

Bhatnagar et al., reported a long fusiform ectopic SCG (right $-3.7 \mathrm{~cm}$; left $-5 \mathrm{~cm}$ ) located at the level of bifurcation of the carotid artery and the elongated ganglion might represent the fusion of SCG and MCG. ${ }^{[9]}$ Mitsuoka et al., studied the branches of SCG and classified the branching pattern into seven types according to the site of origin of branches from the superior, middle or inferior part of the ganglion. ${ }^{[10]}$ In the present report, the SCG extended from the base of the skull to the level of the transverse process of C6 vertebra and the ganglion had the maximum thickness at its upper part on the right side and at the lower part on the left side. The branching pattern was type II (from superior part) and Type IV (from superior and middle part) on the left and right side respectively.

Wisco et al., measured the dimensions of SCG and its distance from the common carotid artery bifurcation in 30 cadavers. They also used pseudocolor heat map to map the SCG and common carotid artery bifurcation. Mean length of the SCG in females and males was $3.81 \mathrm{~cm}$ and $4.11 \mathrm{~cm}$ respectively. The distance from the inferior pole of SCG to common carotid artery bifurcation was $0.41 \mathrm{~cm}$ and $0.29 \mathrm{~cm}$ in females and males respectively. They suggested that common carotid artery bifurcation might be a good landmark for localizing SCG and in cases where stellate ganglion block is contraindicated, SCG block could be an alternative. $^{[11]}$

The safety of the cervical sympathetic chain blockade at the level of SCG to improve the cerebral perfusion was 


\section{Rajmahan et al; Bilaterally Elangated Superiar Cervical Ganglian}

evaluated by Treggiari et al. in nine patients who developed vertebral vasospasm following aneurysmal subarachnoid hemorrhage. This minimally invasive procedure was effective in alleviating mild to moderate symptoms and the treatment could be repeated if necessary. ${ }^{[12]}$ Siegenthaler et al., suggested the ultrasound-guided approach of the SCG to determine the accuracy of the position of the needle in the classical sympathetic blockade. ${ }^{[13]}$ In their pilot study of simulated SCG blocks on 10 cadavers (20 sides), the needletip successfully reached the SCG in 19 sides. Thus, the ultrasound-guided approach had provided a better platform for the ganglionic blockade than the transoral route, thereby avoiding the accidental puncture of the internal carotid artery. ${ }^{[13]}$

SCG might also become enlarged following radiotherapy and mimic a parapharyngeal mass. ${ }^{[14]}$ Yuen et al., reported that a patient developed left-sided Horner syndrome when an enlarged left SCG was mistaken for lymph node metastasis in the retropharyngeal space and removed. ${ }^{[5]}$ Lee et al., explained the necessity of preprocedural imaging of the SCG using 3T-MRI by the intraganglionic hypointensities as an indicator. ${ }^{[2]}$ They identified definite SCG in $73 \%$ of the 93 neck sites studied by MRI. The definitive SCGs were located medial to the internal carotid artery and lateral to longus colli muscle and extended between C2 and C4 vertebrae. Probable SCGs, which were located lateral or posterior to the internal carotid artery and anterior to longus colli muscle, were identified in $27 \%$ of the cases. Thus, using MRI, the SCG can be differentiated from other neck pathologies like retropharyngeal lymphadenopathy. ${ }^{[2]}$

Knowledge about the topographical landmarks of the superior cervical ganglion is an essential road map for the placement of needle during the ganglionic block procedures. ${ }^{[13]}$ This understanding will help in the prevention of injury to the adjacent neurovascular structures. ${ }^{[8,13]}$

\section{Conclusion}

We report a rare case of elongated superior cervical ganglion and the knowledge about such variation is essential to avoid injuries to adjacent vascular structures or its accidental removal when mistaken for a lymphnode. Furthermore, it might serve as a guide for the procedures like sympathetic ganglionic block, cervical spine surgeries, and neck dissections.

\section{References}

1. Standring S. Gray's anatomy: The anatomical basis of clinical practices. 41st Ed. Elsevier Limited, New York. 2016; 468

2. Lee JY, Lee JH, Song JS, Song MJ, Hwang S-J, Yoon RG, et al. Superior Cervical Sympathetic Ganglion: Normal Imaging Appearance on 3T-MRI. Korean Journal of Radiology. 2016;17(5):657

3. Civelek E, Karasu A, Cansever T, Hepgul K, Kiris T, Sabancı A, et al. Surgical anatomy of the cervical sympathetic trunk during anterolateral approach to cervical spine. European Spine Journal. 2008;17(8):991-995

4. Ebraheim NA, Lu J, Yang H, Heck BE, Yeasting RA. Vulnerability of the sympathetic trunk during the anteriorapproach to the lower cervical spine. Spine 2000;25:1603-1606

5. Yuen HW, Goh CH, Tan TY. Enlarged cervical sympathetic ganglion: an unusual parapharyngeal space tumour. Singapore Med J 2006;47:321-323

6. Saylam CY, Ozgiray E, Orhan M, et al. Neuroanatomy of Cervical Sympathetic Trunk: A Cadaveric Study. ClinAnat.2009; 22(3):32430

7. Yin Z, Yin J, Cai J, Sui T, Cao X. Neuroanatomy and clinical analysis of the cervical sympathetic trunk and longuscolli. J Biomed R. 2015;29(6):501-507

8. Kiray A, Arman C, Naderi S, Guvencer M, Korman E. Surgical anatomy of the cervical sympathetic trunk.ClinAnat. 2005;18:179_ 185

9. Bhatnagar KP, Nettleton GS, Kuwabara N, Wagner CE, Campbell FR. A case of bilateral ectopic superior cervical ganglia in man. Annals of Anatomy - AnatomischerAnzeiger. 2003;185(2):149_ 152

10. Mitsuoka K, Kikutani T, Sato I. Morphological relationship between the superior cervical ganglion and cervical nerves in Japanese cadaver donors. Brain and Behavior. 2017;7(2):e00619.

11. Wisco JJ, Stark ME, Safir I, Rahman S. A heat map of superior cervical ganglion location relative to the common carotid artery bifurcation: Anesthesia \& Analgesia. 2012;114(2):462-465

12. Treggiari MM, Romand JA, Martin JB, et al. Cervical sympathetic block to reverse delayed ischemic neurological deficits after aneurysmal subarachnoid hemorrhage.Stroke. 2003; 34:961-967

13. Siegenthaler A, Haug M, Eichenberger U, Suter M R , Moriggl B. Block of the superior cervical ganglion, description of a novel ultrasound-guided technique in human cadavers. Pain Medicine. 2013; 14(5):646-649

14. Kim JM, Kim J, Kim SH, Choi EC. Enlarged superior cervical sympathetic ganglion mimicking a metastatic lymph node in the retropharyngeal space: A case report. Journal of the Korean Society of Radiology. 2017;76(4):278

Copyright: ( $)$ the author(s), publisher. Academia Anatomica International is an Official Publication of "Society for Health Care \& Research Development". It is an open-access article distributed under the terms of the Creative Commons Attribution Non-Commercial License, which permits unrestricted non-commercial use, distribution, and reproduction in any medium, provided the original work is properly cited.

How to cite this article: Rajmohan L, Sakthivel S, Verma S, Kishore A. A Report on Bilaterally Elongated Superior Cervical Ganglion. Acad. Anat. Int. 2019;5(1):87-89.

DOI: dx.doi.org/10.21276/aanat.2019.5.1.20

Source of Support: Nil, Conflict of Interest: None declared. 